\title{
The Regulation of Clinical Trials in Iran - Challenges and Limitations
}

\author{
Zahra Karimian ${ }^{1,2 *}$ \\ ${ }^{1}$ Department of Clinical Pharmacy, School of Pharmacy, International Campus - Iran University of Medical Sciences (Current), Iran \\ ${ }^{2}$ Clinical Pharmacy Specialist and Research Scholar, Iranian Food and Drug Administration - Ministry of Health and Medical Education (Former), Iran
}

The regulation of clinical trials has always been an important responsibility of regulatory authorities, however, with the recent surge in the number of clinical trials investigating treatments and vaccines for the Coronavirus disease 2019 (COVID-19) across the world, oversight and monitoring over clinical trial investigators and sponsors has become increasingly important.

The Iranian Food and Drug Administration (IFDA) is the national regulatory authority under the Ministry of Health and Medical Education (MOHME) which is responsible for protecting public health through ensuring the safety and efficacy of food, drugs, cosmetics and medical devices [1]. The Clinical Trials Center (CTC) in Iran was first established within the IFDA in 2003 to regulate the proper conduction of Clinical Trials (CTs) according to the World Health Organization (WHO) guidelines for Good Clinical Practice (GCP) [2]. Since then, the responsibilities of the CTC have expanded considerably beyond reviewing and approving CT protocols to evaluating post-marketing surveillance studies and periodic safety update reviews, as well as providing training relevant to GCP standards and CT regulations in Iran [3].

As part of the IFDA's mission towards ensuring efficiency and quality of services rendered by the departments within its purview, the performance of the CTC is regularly evaluated by administrators through internal measures and external metrics. However, there are certain processes that have remained largely unchanged since they were initially adopted, which require re-evaluation and comprehensive reform.

Inspections and Audits - While the sponsors are responsible for regularly auditing the CT investigators and centers, the CTC may take part in periodic inspections where trial documentations and records are inspected by a multidisciplinary team, consisting of a regulatory officer from the IFDA, at least one medical expert specialized in a field related to the trial, and an epidemiologist [4]. According to the GCP guidelines, inspections may be announced to the investigators or unannounced. The CTC almost entirely conducts informed inspections; maintaining the position that pre-informed visits are more conducive to capacity-building among investigators, as they are perceived as less scrutinizing and negative [5]. This is while unannounced inspections may be necessary under certain circumstances, such as when there is suspicion of misconduct in a trial - which is unfortunately more common than anticipated [6]. As a result, more impromptu audits by CTC inspectors are warranted in order to identify any irregularities that may temporarily be remedied for with prior knowledge of a regulatory inspection.

Informed Consent Forms and Insurance Policies - Inadequate or delinquent informed consent from participants remains a major area of challenge in Iran. As a result, patients are often unaware of their full rights when enrolled in a trial and cannot discern which health expenditures they have the right to refuse. Randomly contacting trial subjects to ensure that they fully comprehend their entitlements while in a trial seems to be a reasonable first step in identifying investigator noncompliance with this policy. Another matter which warrants re-evaluation by CTC regulators is health insurance coverage for trial subjects. The guidelines issued by the Council for International Organizations of Medical Sciences (CIOMS) in collaboration with the WHO note that proper health insurance coverage should be provided to participants for treatment and compensation of inevitable researchrelated risks or medical injuries that may ensue during the trial [7]. There are currently a few health insurance companies in Iran which offer coverage plans for patients in clinical trials, however, they vary considerably depending on the insurer, CT, and sponsor(s). The CTC in collaboration with national health insurance organizations could look into adopting a more standardized insurance policy system by sponsors and investigators, which could help patients benefit from more uniform compensation policies.

National Clinical Trials Registry - The Iranian Registry of Clinical Trials (IRCT) is part of the WHO Registry Network where investigators are required to register trials prospectively [8]. While similar to ClinicalTrials.gov (USA) and EudraCT (EU) any changes to the original protocol are trackable in the registry, reporting and recording trial results in the registry are not mandatory. Since public access to trial results through registries will help promote the fulfillment of ethical obligations to participants, ensure the overall contribution of research to medical knowledge, and reduce publication and outcome reporting biases $[9,10]$, the CTC should strongly consider mandating the reporting of trial results upon their completion to the IRCT - at least for future protocol authorizations.

Robust Clinical Trials Portal - To date, the CTC has mainly employed the expertise of Iranian clinicians and researchers in the process of evaluating CTs. Exploring the possibility for developing a more robust online system (portal) which could allow international reviewers to participate in the process of assessing and monitoring trial documents and reports will help expand the reviewer network to a wider and more diverse group of medical experts, while providing an

*Correspondence to: Zahra Karimian, PharmD, MPH, Department of Clinical Pharmacy, School of Pharmacy, International Campus of Iran University of Medical Sciences, Iran, E-mail: karimian@umn.edu / karimian.z@iums.ac.ir

Received: August 03, 2020; Accepted: August 11, 2020; Published: August 14, 2020 
opportunity for Iranian clinicians to collaborate in more international (multi-center) CTs.

Assuring the safety of trial participants, maintaining adherence to GCP guidelines, and minimizing risks to trial integrity remain crucial responsibilities of regulators at the IFDA, especially during the COVID-19 public health crisis. Tighter regulation over CTs in Iran will be necessary to address the aforementioned challenges and shortcomings. Allocating additional resources (human and financial) to meet the surge in the number of trials investigating potential treatments and vaccines against COVID-19, along with adopting a more transparent and well-defined legal framework for managing errors, misconduct or fraud detected in the conduction of CTs could facilitate this process.

\section{Declarations}

\section{Funding}

None.

\section{Conflicts of interest}

None.

\section{Acknowledgements}

I wish to acknowledge and express my gratitude to Dr. Rassoul Dinarvand, Deputy to the Minister of Health and Medical Education and former Iranian Food and Drug Administration (IFDA) for the opportunity to serve at the IFDA under his supervision.

\section{References}

1. Iran Food and Drug Administration (2018) The Official Webpage for the IFDA Ministry of Health and Medical Education.

2. WHO (2005) Handbook for good clinical research practice (GCP): guidance for implementation? World Health Organization.

3. Hosseini SAR, Juibary AG (2012) Clinical trials in Iran; biannual report of Clinical Trial Committee in Food and Drug Organization, Ministry of Health and Medical Education of Iran. Arch Iran Med 15: 52--54.

4. Hosseini SAR, Darbooy S, Salimi A (2013) Regulatory aspects of clinical trials in iran third year report of clinical trial committee in food and drug organization. Iran J Public Health 42: 102-106. [Crossref]

5. Clinical Trials Center of the IFDA (2015) Regulatory GCP Inspection - Current Practice in Iran.

6. United States Food and Drug Administration (2010) Office of Good Clinical Practice. FDA Inspections of Clinical Investigators: Information Sheet Guidance For IRBs, Clinical Investigators, and Sponsors.

7. WHO (2017) International ethical guidelines for health-related research involving humans. World Health Organization (WHO) \& Council for International Organizations of Medical Sciences (CIOMS).

8. IRCT (2003) Iran University of Medical Sciences. Iranian Registry of Clinical Trials.

9. Zarin DA, Keselman A (2007) Registering a Clinical Trial in ClinicalTrials.gov. Chest 131: 909-912. [Crossref]

10. https://clinicaltrials.gov/ct2/manage-recs/background

Copyright: $(2020$ Karimian Z. This is an open-access article distributed under the terms of the Creative Commons Attribution License, which permits unrestricted use, distribution, and reproduction in any medium, provided the original author and source are credited. 